\title{
Hydrogenation dynamics of Ru capped $Y$ thin films
}

Cite as: J. Appl. Phys. 126, 145301 (2019); doi: 10.1063/1.5094592

Submitted: 4 March 2019 . Accepted: 22 September 2019.

Published Online: 8 October 2019

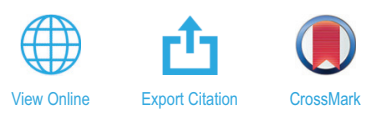

O. Soroka, , ,a) (D) J. M. Sturm, ${ }^{7}$ R. W. E. van de Kruijs, ${ }^{1}$ I. A. Makhotkin, ${ }^{1}$ K. Nikolaev, ${ }^{1}$ (D) S. N. Yakunin, ${ }^{2}$ C. J. Lee, ${ }^{3}$ and F. Bijkerk'

\section{AFFILIATIONS}

${ }^{1}$ Industrial Focus Group XUV Optics, MESA+ Institute for Nanotechnology, University of Twente, Enschede 7500 AE, The Netherlands

${ }^{2}$ National Research Center "Kurchatov Institute," Moscow 123182, Russian Federation

${ }^{3}$ Institute of Engineering, Fontys Hogescholen, Eindhoven 5612 MA, The Netherlands

a) Author to whom correspondence should be addressed: o.soroka@utwente.n|

\begin{abstract}
The structural changes in Ru-coated Y films during hydrogenation were studied in this work. In situ XRD data were used to show that the $\mathrm{Y}$ to $\mathrm{YH}_{2}$ transition requires significant hydrogen loading of the $\mathrm{Y}$ lattice. By comparing the XRD data with the in situ spectroscopic ellipsometry data, an effective medium model for the transition was obtained. This model describes the $\mathrm{Y}$ to $\mathrm{YH}_{2}$ transition well. The $\mathrm{YH}_{2}$ to $\mathrm{YH}_{3}$ transition is also described by an effective medium model, however, with reduced accuracy around the midpoint of the transition. By comparing the $\mathrm{YH}_{2}$ and $\mathrm{YH}_{3}$ crystal sizes, we show that these deviations may be due to a surface plasmon resonance. The improved understanding of the ellipsometry measurements is important for optical hydrogen sensing applications.
\end{abstract}

Published under license by AIP Publishing. https://doi.org/10.1063/1.5094592

\section{INTRODUCTION}

When hydrogenation of thin layers of $\mathrm{Y}$ was studied for the first time, metal-insulator switching during the transition of $\mathrm{YH}_{2}$ to $\mathrm{YH}_{3}$ was discovered. ${ }^{1}$ This optical change upon hydrogenation has found application in many fields, but with a particular emphasis on hydrogen sensing. Many Y-based sensors have been developed for hydrogen gas detection ${ }^{2-4}$ and the measurement of hydrogen diffusion in metals. ${ }^{5}$ These sensors have the advantage of using optical techniques to monitor the change in the hydrogen concentration. This avoids the necessity for electrical connections to the sensor and associated safety risks in the presence of oxygen-hydrogen mixtures. ${ }^{6,7}$

A common feature of yttrium-based $\mathrm{H}$-sensors is the presence of a Pd protective layer. In addition to protecting the yttrium from oxidation, the Pd coating adsorbs and dissociates molecular hydrogen, which is then released as atomic hydrogen into the $\mathrm{Y}$ film. It is possible to reach the $\mathrm{YH}_{3}$ phase in a $\mathrm{Pd} / \mathrm{Y}$ stack by varying the applied hydrogen pressure, though it dissociates back to a stable $\mathrm{YH}_{2}$ phase when the hydrogen supply is switched off. ${ }^{8}$ This happens because (i) the $\mathrm{YH}_{2}$ phase is thermodynamically more stable than $\mathrm{YH}_{3}{ }^{9}$ and (ii) the desorption temperature of hydrogen from the $\mathrm{Pd}$ surface is close to room temperature. ${ }^{10}$ In a previous study, we demonstrated that a $\mathrm{Ru}$ protective layer, with a higher hydrogen desorption temperature, can stabilize $\mathrm{YH}_{3}$ at lower applied hydrogen flux and/or pressure. In order to exploit both the $\mathrm{Y}$ to $\mathrm{YH}_{2}$ and $\mathrm{YH}_{2}$ to $\mathrm{YH}_{3}$ transitions for sensing hydrogen at lower pressures, it, therefore, can be an advantage to use $\mathrm{Ru}$ as a protective material. In addition, it is also desirable to study hydrogen diffusion through layers of other materials. In the case of a Ru protective cap layer, however, an atomic hydrogen source is needed to achieve hydrogenation of Y. Atomic $\mathrm{H}$ reduces native $\mathrm{RuO}_{2}$ on the $\mathrm{Ru}$ surface, which would otherwise inhibit hydrogen diffusion. In this article, we analyzed the hydrogenation of a Y film, covered by a Ru layer, which is exposed to a flux of atomic hydrogen.

Ruthenium coated yttrium films are used to understand the optical properties of $\mathrm{Y}$ as a function of hydrogen loading, structural changes during the hydrogenation and dehydrogenation processes, and thermodynamics and kinetics of $\mathrm{Y}$ hydrogenation. In the previous work, ${ }^{10}$ it was shown that the $\mathrm{YH}_{3}-\mathrm{YH}_{2}$ transition is strongly influenced by the surface binding energy of hydrogen on the surface of the protective material on top of the Y film. The optical properties of the sensor may also be influenced by, for instance, the crystallinity of the different $\mathrm{Y}$ (hydride) phases and lattice expansion.

In most cases, the hydrogen concentration in a $\mathrm{Y}$ film is obtained by measuring its transparency. ${ }^{11,12}$ However, this requires a transparent substrate, reducing the optical contrast between the $\mathrm{YH}_{3}$ film and the substrate near saturation. The lack of contrast between the film and its substrate complicates extracting the film's dielectric 
constants, which leads to increased uncertainty in the hydrogen concentration. To resolve these difficulties, spectroscopic ellipsometry (SE) could be used, which is known to be highly sensitive to the optical properties of both dielectric and metallic films. Therefore, SE is an ideal candidate to increase the sensitivity to hydrogen diffusion for hydrogenation states up to $\mathrm{YH}_{2}$ (where yttrium hydride is metallic), as well as to increase the accuracy of quantification during the $\mathrm{YH}_{2}$ to $\mathrm{YH}_{3}$ transition. Furthermore, by using a high-contrast substrate such as silicon, the optical properties of $\mathrm{YH}_{3}$ near saturation are easier to obtain.

Though there are numerous works on Y hydrogenation, ${ }^{8,13-16}$ only a few of them used ellipsometry to monitor the process. ${ }^{8,16}$ To our knowledge, none of the published data include the dynamics of the phase transition, but instead focus on the beginning and end points of hydrogenation. For a sensor application, however, it is important to understand the structural changes in the $\mathrm{Y}$ film during the hydrogenation process and their impact on the ellipsometry signal.

One possible reason for the lack of dynamic ellipsometry data is that analysis is complicated by multiple processes occurring in parallel. It is difficult to obtain a realistic solution to the inverse problem without a reliable model containing film thicknesses and dielectric constants of the layers in the sample. To address this challenge, we combine in situ SE with ex situ and in situ X-ray diffraction (XRD), and ex situ X-ray reflectivity (XRR) to obtain a detailed picture of the film's structural changes. The combination of in situ and ex situ techniques allows data to be captured during hydrogen in-diffusion and during the phase transformations from $\mathrm{Y}$ to $\mathrm{YH}_{2}$ and $\mathrm{YH}_{2}$ to $\mathrm{YH}_{3}$.
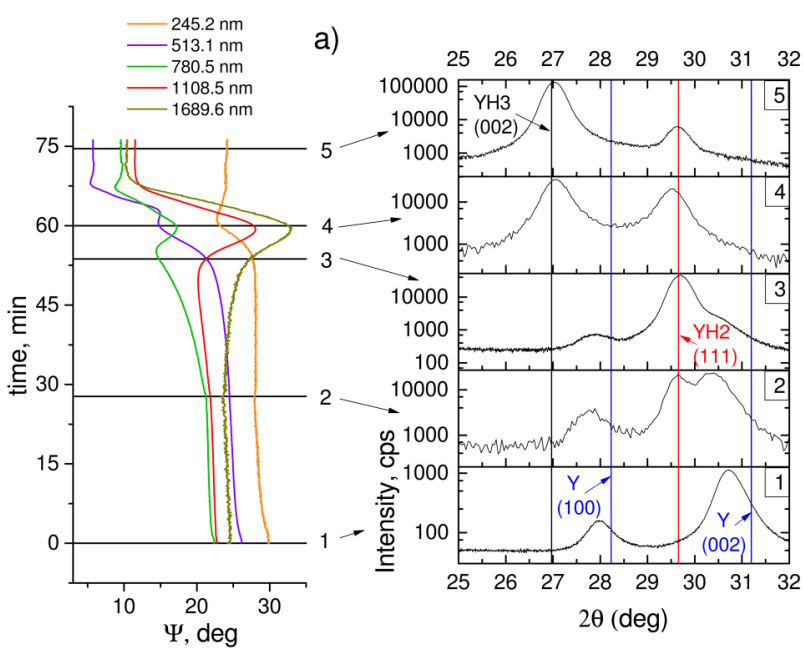

FIG. 1. Time evolution of the ellipsometric angle $\Psi$ for a $70 \mathrm{~nm} Y$ film, coated with $3 \mathrm{~nm}$ of $\mathrm{Ru}$, during exposure to atomic $\mathrm{H}$ [time axis is vertical for ease of comparison to subfigure (b)]. The five displayed wavelengths are evenly distributed over the detected spectrum. Five identical samples were exposed to hydrogen until the indicated times in graph (a). The ex situ XRD spectrum for each sample is shown in graph (b), starting with an unexposed sample (1) and finishing with a maximally saturated sample (5).
In this work, an ellipsometric model is developed for the entire $\mathrm{Y}$ hydrogenation process. Based on the XRD and XRR data, the optical signature of $\mathrm{YH}_{2}$ and $\mathrm{YH}_{3}$ formation was identified in the ellipsometry measurements. This allows for the construction of an accurate ellipsometric model for the transition from $\mathrm{Y}$ to $\mathrm{YH}_{2}$, and a qualitatively accurate model for the $\mathrm{YH}_{2}$ to $\mathrm{YH}_{3}$ transition. The combination of methods reveals insight into why the $\mathrm{Y}-\mathrm{YH}_{2}$ phase transition is delayed with respect to the start of hydrogen exposure. In addition, a possible local surface plasmon resonance in the $\mathrm{YH}_{2}-\mathrm{YH}_{3}$ transition is revealed, which limits the accuracy of our present model to describe the $\mathrm{YH}_{2}-\mathrm{YH}_{3}$ transition.

\section{METHODS}

To study Y hydrogenation, a series of samples were prepared using DC magnetron sputtering in a vacuum chamber with a base pressure of $10^{-8}$ mbar. Si (100) single crystal substrates of $15 \times 15 \mathrm{~mm}^{2}$ size were coated with $70 \mathrm{~nm}$ of $\mathrm{Y}$ and $3 \mathrm{~nm}$ of Ru using Ru and Y targets with a purity of $99.95 \%$. The surface roughness and sample structure were characterized by atomic force microscopy (AFM) (Bruker, Dimension Edge) and XRR (Malvern PANalytical Empyrean).

Hydrogenation of $\mathrm{Y}$ was monitored by in situ spectroscopic ellipsometry (Woollam M-2000XI) at an angle of incidence of about $75^{\circ}$ and a spectral range of $240-1600 \mathrm{~nm}$. Samples were exposed to atomic hydrogen in a vacuum chamber with a base pressure of $2 \times 10^{-8} \mathrm{mbar}$. Hydrogen species were generated by passing $100 \mathrm{sccm}$ of $\mathrm{H}_{2}$ over a W filament heated to $2000^{\circ} \mathrm{C}$, which was placed at $4 \mathrm{~cm}$ from the sample surface. The temperature of the filament was measured using an infrared temperature sensor (Raytek, RayMR1SCCF). The sample temperature was maintained below $40^{\circ} \mathrm{C}$ with a water-cooled sample holder. The hydrogen flux increased the chamber pressure to $2 \times 10^{-2}$ mbar during exposures. The flux to the sample surface was calculated to be $10^{18} \mathrm{at} /\left(\mathrm{cm}^{2} / \mathrm{s}\right)$ after measuring the etch rate of a carbon layer (following the method of Braginsky et al. ${ }^{17}$ ). Atomic hydrogen exposure efficiently reduces the native oxide of the $\mathrm{Ru}$ cap of the $\mathrm{Y}$ film. ${ }^{18}$ Therefore, it is expected that the $\mathrm{Ru}$ oxide film is fully reduced during measurements of the hydrogenation of Y. Maximum hydrogenation was assumed to be achieved when the ellipsometric angles $\Psi$ and $\Delta$ restabilized after a rapid and large change.

For the first set of ellipsometry measurements, exposure to atomic hydrogen was stopped at various moments during hydrogenation $(28,53$, and $59 \mathrm{~min})$. The exposed samples were removed from the vacuum, and XRD (Malvern PANalytical Empyrean) was used to obtain the crystalline structure of the yttrium/yttrium hydride layer. XRD measurements were performed in a $\theta-2 \theta$ geometry using $\mathrm{Cu}-\mathrm{K} \alpha$ radiation $(0.154 \mathrm{~nm})$. Since the $\mathrm{Ru}$ capping layer stabilizes the $\mathrm{YH}_{3}$ phase, it is possible to perform ex situ XRD on partially and fully saturated samples.

In situ XRD measurements during hydrogenation and dehydrogenation were performed using the BM 25 (SpLine) beamline at the European Synchrotron Radiation Facility (ESRF) in Grenoble. A small vacuum chamber (base pressure $6 \times 10^{-6} \mathrm{mbar}$ ) was installed at the first focus point of branch B. ${ }^{19}$ XRD was performed using a photon energy of $20 \mathrm{keV}$. In situ measurements were made in a $\theta-2 \theta$ 
geometry, using a scan range that encompassed the Y (100), Y (002), YH2 (111), and YH3 (002) peaks. Each scan took approximately $3 \mathrm{~min}$. Also, in-plane Grazing Incidence x-ray Diffraction (GIXRD) measurements with a fixed incident angle (higher than the critical angle) were performed in the same $2 \theta$ range. Similar to the ellipsometry measurements, hydrogen exposures were performed by flowing molecular hydrogen past a W filament, placed $4 \mathrm{~cm}$ from the sample surface. To ensure that hydrogenation was slow compared to the scan time, the chamber pressure during exposure was limited to $5 \times 10^{-3} \mathrm{mbar}$ by reducing the hydrogen flow. The filament temperature was set to $1850^{\circ} \mathrm{C}$ using a pyrometer (MAURER, KTR 1075-1-L). Dehydrogenation of $\mathrm{YH}_{3}$ was achieved by switching off the hydrogen supply and heating the sample with a heater that was built into the sample holder.

\section{RESULTS}

\section{A. Ellipsometry and ex situ XRD}

The typical time evolution of the ellipsometric angle $\Psi$ for a $\mathrm{Ru} / \mathrm{Y}$ sample is shown in Fig. 1(a). The ellipsometry data are compared to the XRD data obtained from a set of identical samples that were partially exposed. This comparison is used to determine the intermediate states of $\mathrm{Y}$ hydrogenation. The exposure times indicated by the lines marked 1 through 5 correspond to the times when the exposure to hydrogen of the different samples in the set was interrupted. The as-deposited sample corresponds to state 1 , and state 5 is the maximally hydrogenated sample. The XRD spectra show that the observed changes in $\Psi$ are indeed caused by the formation of $\mathrm{YH}_{2}$ and $\mathrm{YH}_{3}$ [Fig. 1(b)]. The fully hydrogenated sample still has a small fraction of the $\mathrm{YH}_{2}$ phase [subplot 5 of Fig. 1(b), note the logarithmic scale], which was observed in prior research. ${ }^{20}$ The integrated intensity of the $\mathrm{YH}_{2}$ (111) peak for a maximally hydrogenated sample is only $7 \%$ of the maximum integrated intensity of the $\mathrm{YH}_{2}$ (111) and $\mathrm{YH}_{3}(002)$ peaks, which corresponds to $\mathrm{YH}_{2.9}$. This estimate is somewhat higher than the values reported elsewhere, possibly due to the stabilizing effect of the Ru layer. ${ }^{10}$ Once formed, the $\mathrm{YH}_{3}$ phase stays stable under the $\mathrm{Ru}$ cap at the room temperature. Note that the location of the $\mathrm{Y}$ peaks of the as-deposited sample is shifted, which is a result of the film stress.

\section{B. In situ XRD}

In order to understand the formation and dissociation of the $\mathrm{YH}_{3}$ phase, $\mathrm{Ru}$-capped samples were investigated in situ with XRD at SpLine, ESRF. The evolution of the X-ray diffraction pattern during hydrogenation [Fig. 2(a)] and dehydrogenation [Fig. 2(b)] was recorded. Hydrogenation is started by switching the $\mathrm{W}$ filament on. Due to vicinity to the $\mathrm{W}$ filament, the sample was also heated to about $340 \mathrm{~K}$ during hydrogenation, which is indicated below the $2 \theta$ plot of Fig. 2(a). Regardless of the different temperatures and hydrogen pressures, the $\mathrm{Y}$ hydrogenation recorded with in situ XRD [Fig. 2(a)] and SE [Fig. 1(a)] have similar trends in $2 \theta$ peaks shifts and SE angle evolution, respectively. The ex situ XRD measurements show that there is some remaining $\mathrm{YH}_{2}$ at the end of the transition [Fig. 1(b), subplot 5], while a complete transition to $\mathrm{YH}_{3}$ is seen in the in situ experiment. The difference in end hydrogenation states is caused by some release of hydrogen during transportation of samples in air from the $\mathrm{H}$ exposure chamber to the diffractometer.

Following saturation, the temperature was gradually increased from $300 \mathrm{~K}$ until dehydrogenation started, which corresponded to a temperature of $410 \mathrm{~K}$ [see Fig. 2(b)]. As discussed previously, ${ }^{10}$ this temperature is an activation temperature for the desorption of hydrogen from the Ru surface. Note that the phase transition from $\mathrm{YH}_{2}$ to $\mathrm{YH}_{3}$ occurs faster than the reverse transition $\{5 \mathrm{~min}$ for hydrogenation [Fig. 2(a)], compared to $20 \mathrm{~min}$ for dehydrogenation [Fig. 2(b)]\}, even when the temperature is above the threshold temperature. A similar slower speed of dehydrogenation compared to hydrogenation has been observed in Pd-capped Y systems and was attributed to stress in the film and the kinetics of structure transformations. ${ }^{15}$ However, for Ru-capped Y films, the lower rate of hydrogenation is not only due to film stress but also due to the higher surface desorption temperature of hydrogen from ruthenium. ${ }^{10,21}$

As hydrogenation begins, the lattice slowly expands, but there is no evidence of $\mathrm{YH}_{2}$ formation. This is clearly visible in Fig. 2(a) where from 0 to $20 \mathrm{~min}$ the Y (002) XRD peak shifts from $12.26^{\circ}$ to $12.05^{\circ}$. Such a shift is significantly larger than expected from thermal expansion (heating by $40 \mathrm{~K}$ would result in a $2 \theta$ shift of $0.005^{\circ}$ ), and is most likely caused by the presence of interstitial hydrogen expanding the $\mathrm{Y}$ lattice. Thus, crystalline $\mathrm{YH}_{2}$ formation takes place after the $\mathrm{Y}$ lattice is at least partially loaded with hydrogen. However, the enthalpy of formation for $\mathrm{YH}_{2}$ is negative and the applied hydrogen pressure and flux of atomic $\mathrm{H}$ are sufficient to be above the plateau pressure for saturating the $\mathrm{Y}$ film to $\mathrm{YH}_{3}$ in equilibrium conditions, implying that $\mathrm{YH}_{2}$ should form continuously.

It is likely, however, that the formation of isolated $\mathrm{YH}_{2}$ in a Y crystal structure is energetically unfavorable due to the increase in the surface energy of both $\mathrm{Y}$ and $\mathrm{YH}_{2}$ at the interface formed between the two. On the other hand, the energy in the film increases due to the strain imposed by lattice expansion. Hence, the transition from $\mathrm{Y}$ to $\mathrm{YH}_{2}$ only begins when the increase in energy due to the elastic strain exceeds the increase in energy due to the interface formed between $\mathrm{Y}$ and $\mathrm{YH}_{2}$. The strain energy of the $\mathrm{Y}$ lattice is used to estimate a pseudoactivation energy for the formation of the $\mathrm{Y} / \mathrm{YH}_{2}$ interface. The elastic energy equals $U=\frac{1}{2} E\left(\frac{\Delta d}{d}\right)^{2}$, where $E$ is Young's modulus, $E=63.5 \mathrm{GPa}^{22} d$ is the initial lattice constant of $\mathrm{Y}$, and $\Delta d$ is the difference in the $\mathrm{Y}$ lattice constants between the beginning of exposure and before the $\mathrm{YH}_{2}$ formation. A calculation yields an elastic energy of $2.1 \mathrm{eV} /$ at. along the (002) diffraction plane. This implies $2.1 \mathrm{eV}$ for the interface formation between $\mathrm{Y}$ and $\mathrm{YH}_{2}$.

Since the enthalpy of $\mathrm{YH}_{2}$ formation is $-2.25 \mathrm{eV} /$ at. $\mathrm{H},{ }^{23}$ the phase transition proceeds rapidly once the energy stored in the lattice is sufficient to initiate the transition. The enthalpy of $\mathrm{YH}_{3}$ formation is only slightly lower at $-2.54 \mathrm{eV} / \mathrm{at}^{23}$ The formation of the $\mathrm{YH}_{3}$ phase is twice as fast as for $\mathrm{YH}_{2}$ with no evidence of lattice expansion: it only takes about $6 \mathrm{~min}$, compared to $12 \mathrm{~min}$ for the $\mathrm{Y}$ to $\mathrm{YH}_{2}$ transition (not including the time of $\mathrm{Y}$ lattice expansion). This can be expected when both transitions are limited by $\mathrm{H}$ flux diffusing through $\mathrm{Ru}$ (since half of hydrogen is required for the $\mathrm{YH}_{2}$ to $\mathrm{YH}_{3}$ transition in comparison to $\mathrm{Y}$ to $\mathrm{YH}_{2}$ ). Hence, it can be concluded that diffusion through $\mathrm{Y}, \mathrm{YH}_{2}$, and $\mathrm{YH}_{3}$ is not a rate-limiting step. 

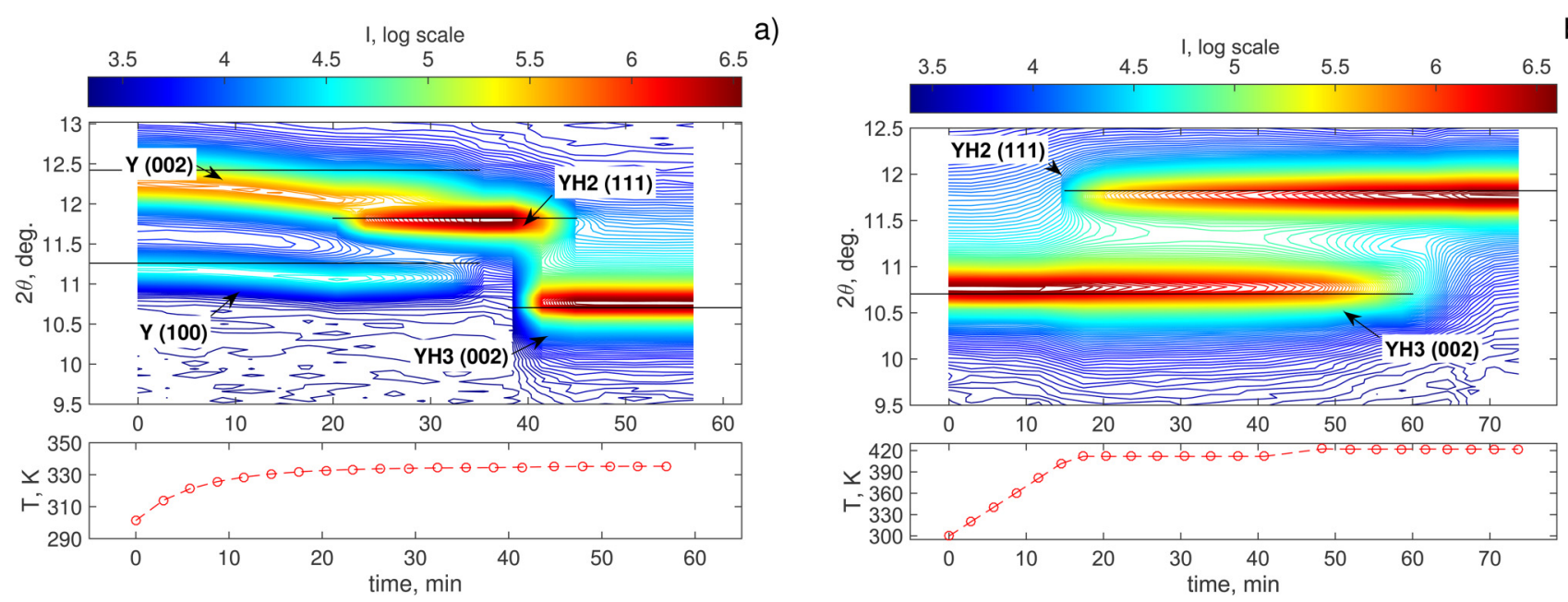

b)

FIG. 2. Time evolution of in situ XRD spectra for a $70 \mathrm{~nm}$ Y film, coated with $3 \mathrm{~nm}$ of Ru, during hydrogenation (a) and dehydrogenation (b). Horizontal lines indicate the tabulated diffraction angles for a $Y$ powder. The lower subplots show the sample temperature during (de)hydrogenation.

It should also be noted that XRD measurements are only sensitive to the presence of crystalline phases. Hence, from XRD alone we cannot exclude the formation of intermediate amorphous $\mathrm{YH}_{2}$ and $\mathrm{YH}_{3}$ phases. Ellipsometry is sensitive to the formation of amorphous phases, because the optical properties of amorphous materials are different from the crystalline polymorphs of the same chemical composition. We discuss the possibility of the existence of amorphous $\mathrm{YH}_{2}$ and $\mathrm{YH}_{3}$ phases in Sec. III C.

To better understand the growth and decay of the crystallites in the film, $\theta-2 \theta$ XRD measurements were complemented with in-plane $2 \theta$ Grazing Incidence $x$-ray Diffraction (GIXRD), the combination of which allowed probing the atomic planes in two orthogonal directions: in-plane and normal to the sample surface. To obtain the crystallite size, the XRD peaks were fitted with a pseudo-Voigt function. The extracted parameters, such as the peak position and FWHM, are plotted as a function of time in Figs. 3(a) and 3(b). Since the crystallites in (a) and (b) are probed in perpendicular directions, $\mathrm{YH}_{3}$ peaks that are produced by mutually perpendicular atomic planes, (002) and (100), are observed. It should be noted that, due to the (111) preferred orientation (normal to the sample surface) of the $\mathrm{YH}_{2}$ phase, the $\mathrm{YH}_{2}$ peak is not present in the in-plane GIXRD measurement. Both $\theta-2 \theta$ (a) and GIXRD (b) measurements confirm that the lattice expands in the (002) direction to a greater extent [see Figs. 3(a) and 3(b)], which is in line with earlier neutron scattering experiments. ${ }^{24,25}$ Using the Scherrer equation, ${ }^{26}$ the crystal sizes were calculated from the FWHM values and are shown in Figs. 3(c) and 3(d). The average crystal size rapidly increases after the transition from $\mathrm{Y}$ to a hydride phase.

The XRD data reveal several necessary elements required for an ellipsometry model. The hydrogen loading before the $\mathrm{YH}_{2}$ transition will cause a minor modification in the $\mathrm{Y}$ optical properties. The lack of $\mathrm{YH}_{2}$ lattice expansion indicates that no significant hydrogen loading is required for the $\mathrm{YH}_{3}$ transition. However, it is known that the optical properties of the $\mathrm{Y}-\mathrm{H}$ system strongly change with the hydrogenation state between $\mathrm{YH}_{1.9}$ and $\mathrm{YH}_{2.1}$. Thereby, we take the $\mathrm{YH}_{1.9}$ state as end point of the $\mathrm{Y}-\mathrm{YH}_{2}$ transition as observed by XRD and verify that the ellipsometry spectra can be fitted with literature data of the dielectric function of $\mathrm{YH}_{1.9}$, as will be further detailed in Sec. III C. We will still refer to this phase as $\mathrm{YH}_{2}$ for simplicity.

\section{Ellipsometry modeling}

Let us now focus on the interpretation of the ellipsometry data obtained during hydrogenation. Figure 4 shows the time evolution of the ellipsometric angles for the Ru capped Y film during atomic $\mathrm{H}$ loading. When considering the entire hydrogenation process, we take into account only two processes that lead to a change of the reflectance, namely, the removal of native ruthenium oxide $^{27}$ and the formation of yttrium hydrides. The large optical contrast between $\mathrm{Y}, \mathrm{YH}_{2}$, and $\mathrm{YH}_{3}$ dominates the reflectance change and, once the $\mathrm{Y}$ to $\mathrm{YH}_{2}$ transitions begin, we attribute all changes in the reflectance solely to transformations of the yttrium (hydride) layer. However, due to ruthenium oxide removal and $\mathrm{Y}$ lattice expansion, small changes of reflectance (first $30 \mathrm{~min}$ in Fig. 4) happen before $\mathrm{YH}_{2}$ starts to form. Since ellipsometry is most sensitive to the topmost layer of metal-coated samples, even small changes on the surface of a Ru cap will affect the reflectance. Therefore, the initial removal of $\mathrm{RuO}_{2}$ should not be neglected. The observed $\mathrm{Y}$ lattice expansion is assumed to have a smaller impact on the reflectance and, therefore, is not taken into account by the model.To successfully model the hydrogenation of $\mathrm{Y}$, we divide the process into three ranges (Fig. 4). The thicknesses of $\mathrm{Ru}$ and Y layers were obtained from XRR to be 3 and $68 \mathrm{~nm}$, respectively, and assumed constant throughout first two ranges. The $\mathrm{Y}$ thickness was set as a free parameter for the $\mathrm{YH}_{2}$ to $\mathrm{YH}_{3}$ transition (the 3rd range). When representing a layer as a mixture of phases/materials in each range, an effective medium 


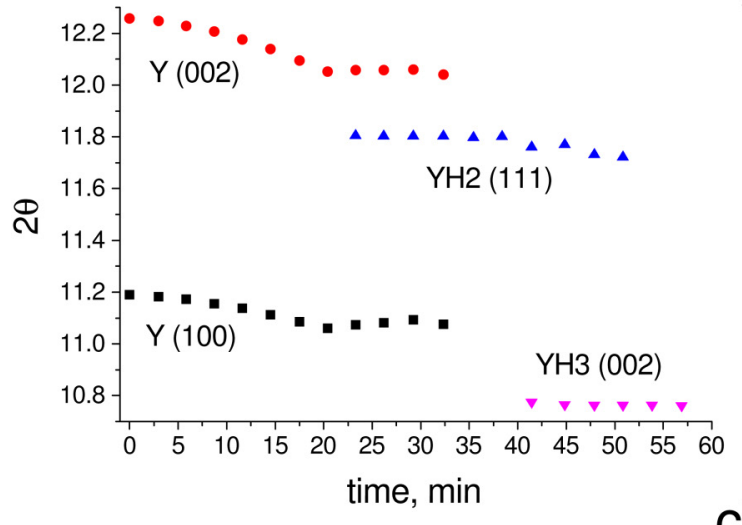

a)
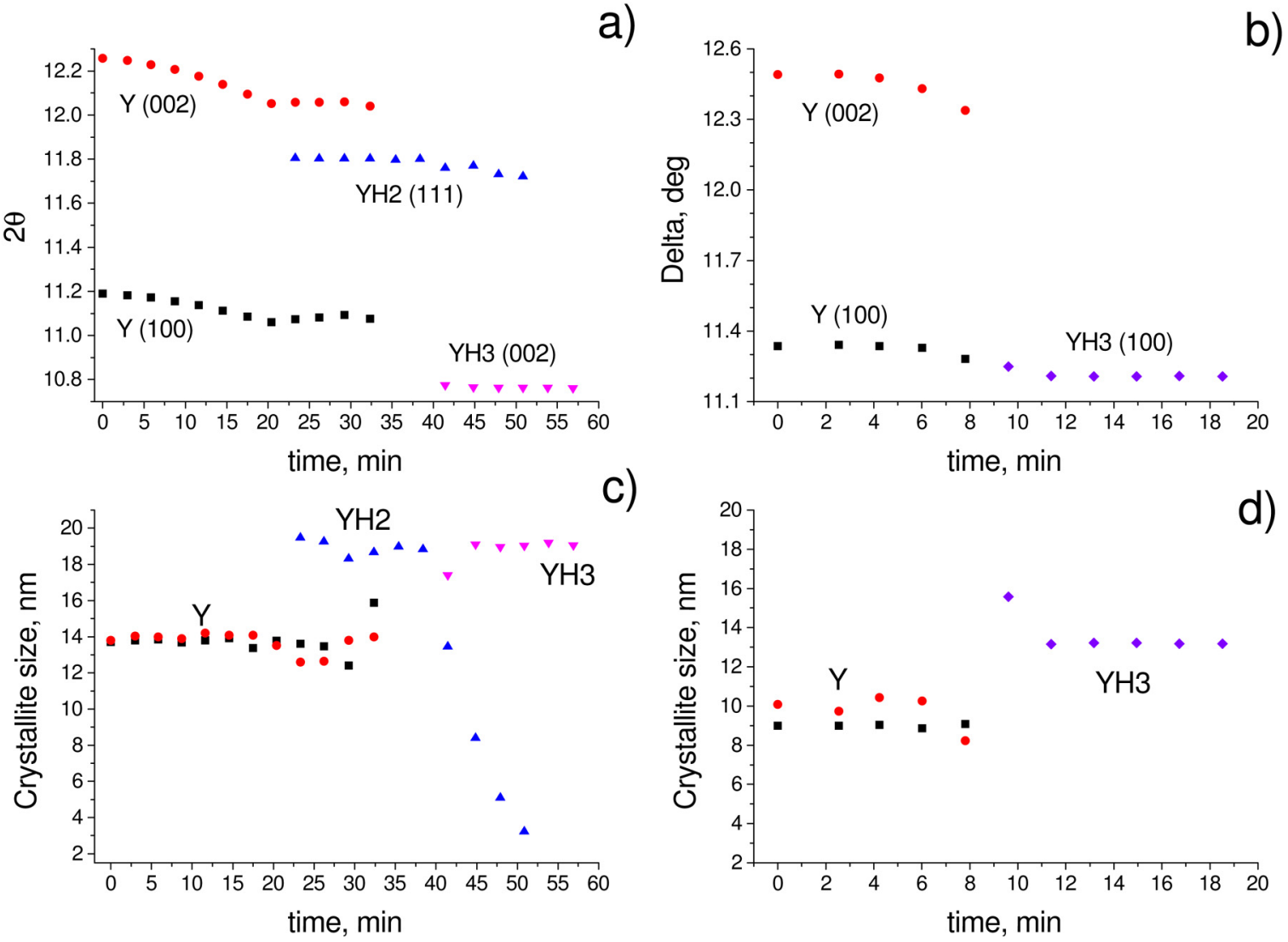

FIG. 3. Time evolution of XRD peak positions for a $70 \mathrm{~nm}$ Y film, coated with $3 \mathrm{~nm}$ of Ru, during hydrogenation in $\theta-2 \theta(a)$ and in the in-plane grazing incidence geometry (b). The time evolution of the crystallite size, calculated from the peak width, is shown in (c) and (d). The time of hydrogenation is different due to the different hydrogen pressures: $[(a)$ and $(c)] 3 \times 10^{-3} \mathrm{mbar}$ and $[(b)$ and $(\mathrm{d})] 1.6 \times 10^{-2} \mathrm{mbar}$.

approximation (EMA) is used in a Bruggeman analysis mode. Optical constants for $\mathrm{RuO}_{2}, \mathrm{Y}$, and $\mathrm{YH}_{2}$ were obtained from prior studies. $^{28,29}$ Van Gogh et al. ${ }^{28}$ have published optical constants for $\mathrm{YH}_{3}$. But due to the lack of contrast between $\mathrm{YH}_{3}$ and the glass substrate in Van Gogh's experiment, the dielectric constants (even when including a non-zero fraction of $\mathrm{YH}_{2}$ ) could not reproduce the SE measurements in this work. Indeed, the variation in maximum hydrogenation between different published results makes it difficult to obtain a reliable optical model for $\mathrm{YH}_{3}$. Hence, we chose not to use published optical models for $\mathrm{YH}_{3}$.

The effective optical constants for $\mathrm{Ru}$ and $\mathrm{YH}_{3}$ were obtained from b-spline fits. It is known that $\mathrm{RuO}_{2}$ is reduced under atomic hydrogen flux, ${ }^{27}$ and we assume that range I in Fig. 4 corresponds mainly to $\mathrm{RuO}_{2}$ reduction. Thus, the end of range I corresponds to a metallic Ru layer.

A b-spline fit to the ellipsometry spectrum, using the model shown in the first row of Table I, yields effective optical constants for $\mathrm{Ru}$. These constants, and the thickness of the Ru layer, are not changed for the remainder of the modeling procedure. After the optical constants for $\mathrm{Ru}$ are obtained, the optical constants for $\mathrm{YH}_{3}$ are obtained by performing the same fitting procedure at the end of the hydrogenation procedure. In this case, we use the model from the second row of Table I. The best fit is obtained for a $\mathrm{YH}_{3}$ thickness of $78 \mathrm{~nm}$, which is consistent with the calculated volumetric lattice expansion of $12 \% .{ }^{10}$ To investigate the reliability of the obtained dielectric function, the b-spline dielectric function for $\mathrm{YH}_{3}$ was then parametrized using the sum of two Tauc-Lorentz oscillators (see Fig. 5). The Tauc-Lorentz oscillator is given by ${ }^{30}$

$$
\begin{gathered}
\varepsilon_{2}= \begin{cases}\frac{A E_{0} B\left(E-E_{g}\right)^{2}}{\left(E^{2}-E_{0}^{2}\right)^{2}+B^{2} E^{2}} \cdot \frac{1}{E}, & \text { where } E>E_{g}, \\
0, & \text { where } E \leq E_{g},\end{cases} \\
\varepsilon_{1}=\frac{2}{\pi} P \int_{E_{g}}^{\infty} \frac{\xi \varepsilon_{n 2}(\xi)}{\xi^{2}-E^{2}} d \xi,
\end{gathered}
$$

where $\varepsilon_{1}$ and $\varepsilon_{2}$ are real and imaginary parts of a dielectric function, respectively, $A$ is the oscillator amplitude, $B$ is the broadening 


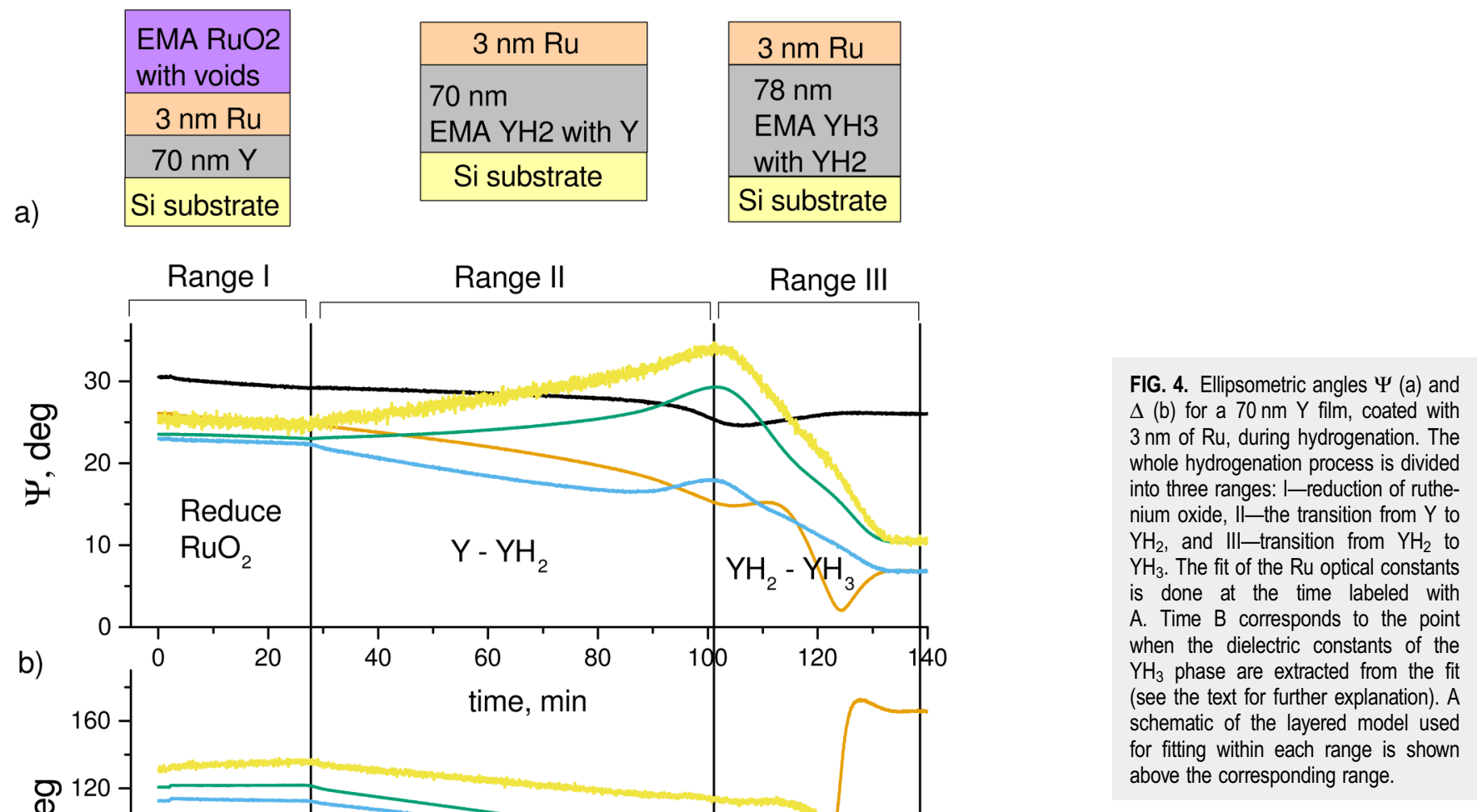

term of the peak, $E_{0}$ is the peak central energy of the oscillator, $E_{\mathrm{g}}$ is the bandgap, and $E$ is the photon energy (all parameters are in electron volt). $P$ denotes the Cauchy principal value of the integral (over the photon energy). Parameterizing the b-spline yields a

TABLE I. Sources of the optical constants of all materials used in the fitting procedure.

\begin{tabular}{ll}
\hline \hline Material & \multicolumn{1}{c}{ Source/model } \\
\hline $\mathrm{Ru}$ & $\begin{array}{l}\text { b-spline fit of } \mathrm{Ru} \text { dielectric constants at point A (see } \\
\text { Fig. 4) }\end{array}$ \\
$\mathrm{YH}_{3}$ & b-spline fit of $\mathrm{YH}_{3}$ optical constants at point B in Fig. 4 \\
$\mathrm{Y}$ & Ref. 28 \\
$\mathrm{YH}_{2}$ & Ref. 28, dielectric function for $\mathrm{YH}_{1.9}$ \\
$\mathrm{RuO}_{2}$ & Ref. 29 \\
\hline \hline
\end{tabular}

bandgap of $2.5 \mathrm{eV}$, which is consistent with values reported elsewhere.

With optical models for the individual components in place, the ellipsometry data are analyzed using EMA models for the full temporal range. The schematics of the layered models and fitting procedures are summarized in Fig. 4. In range I, where ruthenium oxide is removed, an EMA model of $\mathrm{RuO}_{2}$ and voids is used. In range II, where the $\mathrm{Y}$ to $\mathrm{YH}_{2}$ phase transition takes place, an EMA of $\mathrm{Y}$ and $\mathrm{YH}_{2}$ is used. And, finally, in range III, an EMA of $\mathrm{YH}_{2}$ and $\mathrm{YH}_{3}$ is used. In the first two ranges, only one parameter is fit: the ratio of the two relevant constituents (see Table II). The angle offset (the offset of the incident angle) and the thickness of the $\mathrm{YH}_{2} / \mathrm{YH}_{3}$ film are also set as free parameters in the III range. The thickness of the film is expected to expand during the phase transition, so must be fit, while the angle of incidence is allowed to vary to account for the thermal expansion of the mounting system. 


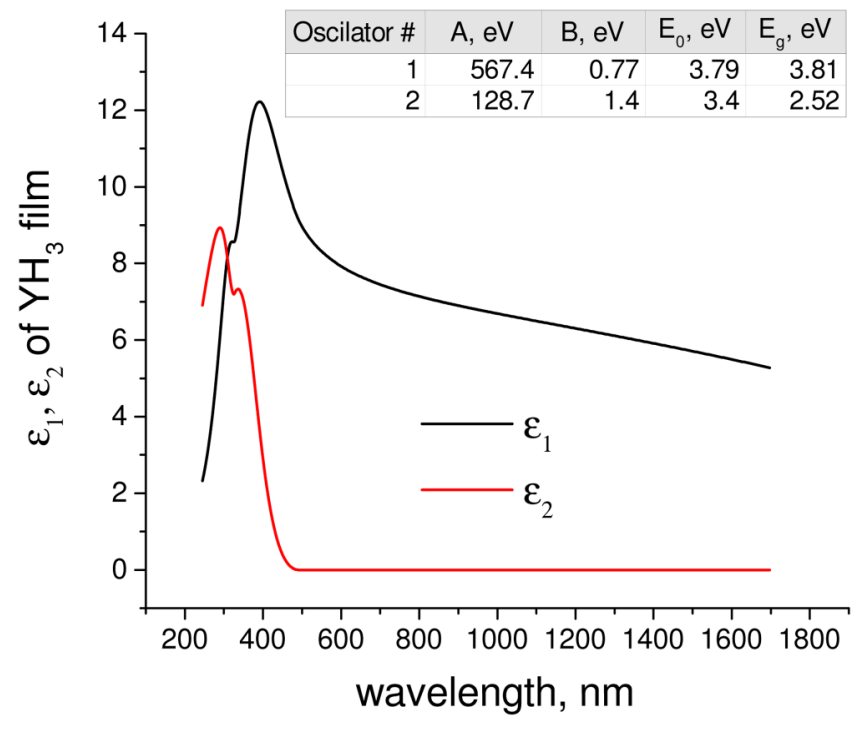

FIG. 5. Parametrized Tauc-Lorentz dielectric function for $\mathrm{YH}_{3}$, extracted from a b-spline fit to the data (see Table I).

Figure 6(a) shows the results of fitting the entire hydrogenation process in the manner described above. More detail regarding ellipsometry measurements and fitting results is provided in the supplementary material. In range $\mathrm{I}$, the fraction of voids in $\mathrm{RuO}_{2}$, which represents the fraction of pinholes in the oxide, rapidly increases when the $\mathrm{W}$ filament is switched on and stays constant thereafter. A void fraction of $100 \%$, which corresponds to an oxidefree surface, was never reached, similar to the data from Ugur et al. ${ }^{18}$ However, an XPS study on the reduction of $\mathrm{RuO}_{2}$ by atomic hydrogen showed that nearly complete reduction can be achieved. ${ }^{31}$ This probably implies that the model does not fully describe the $\mathrm{RuO}_{2}$ reduction process. We speculate that this may be partially caused by substoichiometry of the initial $\mathrm{RuO}_{2}$ film. In addition, simultaneously with the reduction of $\mathrm{RuO}_{2}$, the expansion of the $\mathrm{Y}$ lattice starts, which was omitted in the model used here. Nevertheless, the model consistently shows that the void fraction in $\mathrm{RuO}_{2}$ increases rapidly after atomic hydrogen is supplied, suggesting that oxide reduction happens much faster than $\mathrm{H}$ permeation through the Ru capping layer. Previous research on Ru-capped thin films indicates that the surface roughness of $\mathrm{Ru}$ layers does not

TABLE II. The free parameters of each model that were used for fitting ellipsometric data (see Fig. 4).

\begin{tabular}{ll}
\hline \hline $\begin{array}{l}\text { Fit } \\
\text { range }\end{array}$ & \multicolumn{1}{c}{ Free parameters } \\
\hline I & $\begin{array}{l}\text { Fraction of voids in the } \mathrm{RuO}_{2} \text { layer } \\
\text { II }\end{array}$ \\
III & $\begin{array}{l}\text { Fraction of } \mathrm{Y} \text { in } \mathrm{YH}_{2} \\
\text { Fraction of } \mathrm{YH}_{3} \text { in the } \mathrm{YH}_{2} \text { mixture; Angle offset; layer } \\
\text { thickness }\end{array}$ \\
\hline
\end{tabular}
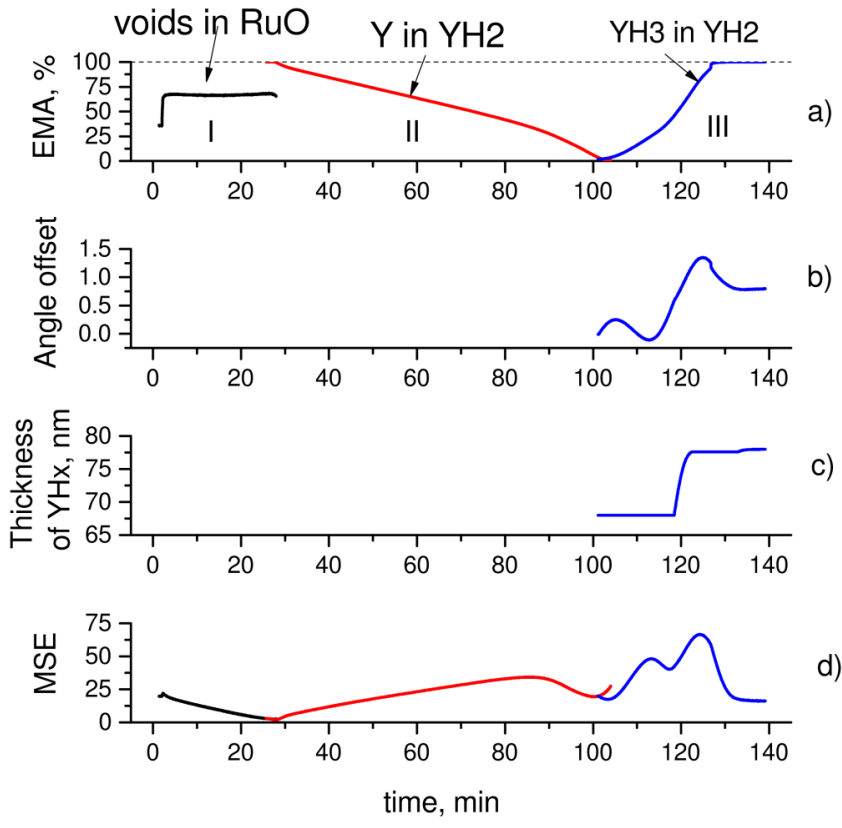

FIG. 6. The fitted fractions (a) and mean square error (d) of the fit as a function of time: range $1-$ fraction of voids in $\mathrm{RuO}_{2}$ layer, range $\|-$ fraction of $\mathrm{Y}$ in $\mathrm{YH}_{2}$ phase, and range III-fraction of $\mathrm{YH}_{3}$ in $\mathrm{YH}_{2}$. The angle offset (b) and the $\mathrm{YH}_{3}$ thickness (c) were also fit parameters in range III.

increase due to atomic hydrogen exposure. ${ }^{31}$ Therefore, we do not consider porosity of the Ru layer in our model.

The hydrogenation trend in range II and range III agrees well with the observations from in situ XRD (Fig. 2). The time of $\mathrm{YH}_{2}$ phase formation (the border of ranges II and III in Fig. 4) was reliably determined from the fitting of ranges II and III. Since range II excludes the sudden change in optical properties between hydrogenation states $\mathrm{YH}_{1.9}$ and $\mathrm{YH}_{2.1}$, a smooth concentration change in range II is obtained. Thus, we conclude that the model, in general, shows the degree of hydrogenation relatively well. The fit of $\mathrm{YH}_{2}$ to $\mathrm{YH}_{3}$ transition was improved to some extent by setting the angle offset and the thickness of $\mathrm{YH}_{\mathrm{x}}$ as free parameters [Figs. 6(b) and 6(c)]. However, the high mean square error in the range III [Fig. 6(d)] suggests that the optical properties of the film are not well described by the model over the entire phase transition.

It is clear from the XRD data that there may be some $\mathrm{YH}_{2}$ still present at saturation, which might introduce some minor additional error. However, including $\mathrm{YH}_{2}$ at $7 \%-10 \%$ concentration in the construction of the optical model does not improve the fit. Therefore, it is most likely that a physical process has not been included in the model. It should be noted that an initial bad fit in range III [Fig. 6(d)] may be due to the change of optical properties between $\mathrm{YH}_{1.9}$ and $\mathrm{YH}_{2.1}$, which was not accounted for in the ellipsometric model.

Wang et al. ${ }^{13}$ have shown that reconstruction of bcc $\mathrm{YH}_{2}$ into hcp $\mathrm{YH}_{3}$ creates voids in the film due to shrinkage of the crystal lattice in the plane perpendicular to the $\mathrm{c}$-axis of the $\mathrm{YH}_{3}$ lattice. 
However, adding voids as a third constituent to the $\mathrm{YH}_{2}-\mathrm{YH}_{3}$ mixture did not improve the fit. Another possibility is the presence of amorphous material. It is possible that there is an unknown fraction of amorphous $\mathrm{YH}_{2}$ or $\mathrm{YH}_{3}$ in the sample during the phase transition, but the end point is highly crystalline. For the case of the $\mathrm{YH}_{2}$ to $\mathrm{YH}_{3}$ phase transition, we attempted to include a third material in the EMA model (with optical properties obtained from a b-spline fit at the point where the MSE was maximum). However, this was not observed to improve the fit substantially. Although not conclusive, the failure to obtain an improved fit suggests that it is unlikely that a significant amorphous phase forms.

The formation of a metal-dielectric nanostructure is known to modify the optical properties of thin films in ways that are not well described by common effective medium theories. ${ }^{32}$ Mie theory describes how the growth of isolated nanocrystals may give rise to plasmonic effects that make an EMA model invalid. ${ }^{33,34}$ When comparing the time at which the maxima in the MSE occur [Fig. 6(d)] with the mean crystal size estimation of $\mathrm{YH}_{2}$ and $\mathrm{YH}_{3}$ by XRD [Fig. 3(c)], it is observed that peaks in the MSE correspond to small isolated $\mathrm{YH}_{3}$ crystallites in a $\mathrm{YH}_{2}$ matrix (in the beginning of range III) and, later, to isolated $\mathrm{YH}_{2}$ crystals in $\mathrm{YH}_{3}$. Mie scattering theory calculations ${ }^{34}$ of $\mathrm{YH}_{2}$ spheres, embedded in a $\mathrm{YH}_{3}$ matrix, reveal that $\mathrm{YH}_{2}$ has a local surface plasmon resonance at $450 \mathrm{~nm}$ (see Fig. 7). This resonance may become significant during $\mathrm{YH}_{3}$ formation at two points during the transition. The resonance may be excited near the beginning of the transition, when small crystallites of $\mathrm{YH}_{3}$ are isolated in $\mathrm{YH}_{2}$. Near the end of the transition, when there are isolated crystallites of $\mathrm{YH}_{2}$ in a $\mathrm{YH}_{3}$ matrix, the resonance may also be excited. In between these two points, the increased connectivity between isolated crystallites will broaden the surface plasmon resonance peak and reduce its influence. Although neither the crystal growth data nor the MSE provides direct evidence for a surface plasmon resonance, the combined data of Figs. 3 and 6 support its existence and effect on the ellipsometry spectrum.

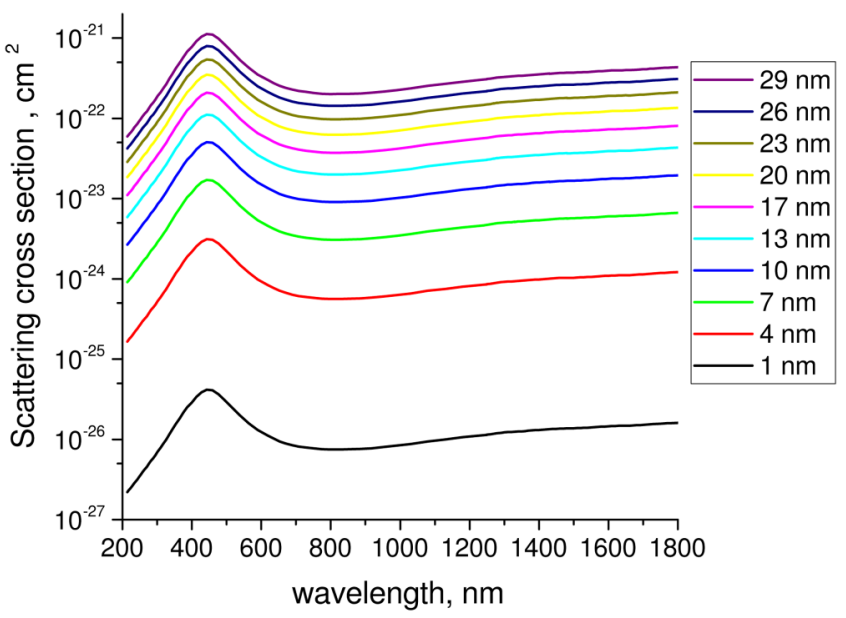

FIG. 7. The scattering cross section of a single isolated $\mathrm{YH}_{2}$ sphere in a $\mathrm{YH}_{3}$ medium, calculated from the Mie scattering theory. ${ }^{34}$

\section{CONCLUSIONS}

In this work, the structural changes of Ru-coated Y films during hydrogenation are characterized. An ellipsometric model was developed to quantify the ratio of hydride phases $\mathrm{YH}_{2}$ and $\mathrm{YH}_{3}$ in a $\mathrm{Y}$ film, from which the absorbed amount of hydrogen can be determined. The proposed model encompasses the entire hydrogenation process, which, in combination with other structure analysis, allowed a consistent study of hydride formation. The results suggest that $\mathrm{RuO}_{2}$ is reduced to $\mathrm{Ru}$ by atomic hydrogen in the first seconds, well before any phase transformation occurs. We show that the delayed onset of the $\mathrm{Y}$ to $\mathrm{YH}_{2}$ transition is not due to the lack of hydrogen in the $\mathrm{Y}$ lattice. Instead, the lattice first loads with hydrogen, followed by a rapid phase transition. We speculate that the delayed onset is because the $\mathrm{Y}-\mathrm{YH}_{2}$ interface energy is rather large at approximately $2.1 \mathrm{eV}$.

Once the transition begins, it is described well by an effective medium approximation using pre-existing optical models for $\mathrm{Y}$ and $\mathrm{YH}_{2}$. The time of $\mathrm{YH}_{2}$ formation was reliably determined from the model, which is an important step for sensor development. The hydrogenation time can be used to estimate the hydrogen flux through the capping layer.

To describe the $\mathrm{YH}_{2}$ to $\mathrm{YH}_{3}$ transition, we obtained an optical model for the $\mathrm{YH}_{3}$ phase, with parameters, such as the bandgap, that are broadly in agreement with previous models. In this case, however, the transition deviates from an EMA model. These deviations occur at stages in the transition when isolated crystallites are present, for which calculations show a significant plasmonic resonance. This can be interesting for future applications of active plasmonics with a tunable size of the $\mathrm{YH}_{2}$ crystallites. For highly accurate hydrogen sensors, a model that includes plasmonic effects may be necessary. For that, additional experiments for isolating the plasmonic effect are desirable.

\section{SUPPLEMENTARY MATERIAL}

See the supplementary material for spectral ellipsometry data and its fit with the EMA models at chosen time points.

\section{ACKNOWLEDGMENTS}

The authors would like to thank Dr. German R. Castro and Dr. Juan Rubio-Zuazo for technical support at ESRF and Mr. Cedric Hendrikx for help in conducting the experiments at ESRF. We also thank Mr. Theo van Oijen for depositing samples and Mr. Goran Milinkovic and Mr. John de Kuster for the technical support. This work is part of the research programme of the Netherlands Organization for Scientific Research (NWO), Domain Applied and Engineering Sciences (AES, previously Technology Foundation STW). This work is additionally supported by ZEISS. We also acknowledge the support of the Industrial Focus Group XUV Optics at the MESA+ Institute at the University of Twente, notably the industrial partners ASML, ZEISS, Malvern Panalytical, and the Province of Overijssel.

\section{REFERENCES}

${ }^{1}$ J. N. Huiberts et al., "Yttrium and lanthanum hydride films with switchable optical properties," Nature 380, 231-234 (1996).

${ }^{2}$ N. Strohfeldt et al., "Yttrium hydride nanoantennas for active plasmonics," Nano Lett. 14, 1140-1147 (2014). 
${ }^{3}$ P. Ngene et al., "Seeing hydrogen in colors: Low-cost and highly sensitive Eye readable hydrogen detectors," Adv. Funct. Mater. 24, 2374-2382 (2014).

${ }^{4}$ F. J. A. A. den Broeder et al., "Visualization of hydrogen migration in solids using switchable mirrors," Nature 394, 656-658 (1998).

${ }^{\mathbf{5}}$ A. Remhof et al., "Switchable mirrors for visualization and control of hydrogen diffusion in transition metals," Phys. Rev. B: Condens. Matter Mater. Phys. 66, 020101 (2002).

${ }^{6} \mathrm{M}$. A. Butler, "Fiber optic sensor for hydrogen concentrations near the explosive limit," J. Electrochem. Soc. 138, L46 (1991).

${ }^{7}$ T. Radeva et al., "Highly sensitive and selective visual hydrogen detectors based on $\mathrm{Y}_{\mathrm{x}} \mathrm{Mg}_{1-\mathrm{x}}$ thin films," Sens. Actuators B Chem. 203, 745-751 (2014).

${ }^{8}$ A. T. M. van Gogh et al., "Structural, electrical, and optical properties of $\mathrm{La}_{1-\mathrm{z}} \mathrm{Y}_{\mathrm{z}} \mathrm{H}_{\mathrm{x}}$ switchable mirrors," Phys. Rev. B: Condens. Matter Mater. Phys. 63, 195105 (2001).

${ }^{9}$ L. N. Yannopoulos, R. K. Edwards, and P. G. Wahlbeck, "The thermodynamics of the yttrium-hydrogen system," J. Phys. Chem. 69, 2510-2515 (1965).

${ }^{10}$ O. Soroka, J. M. Sturm, R. W. E. van de Kruijs, C. J. Lee, and F. Bijkerk, "Control of $\mathrm{YH}_{3}$ formation and stability via hydrogen surface adsorption and desorption,” Appl. Surf. Sci. 455, 70-74 (2018).

${ }^{11} \mathrm{M}$. W. Lee and W. P. Shin, "Optical properties of the $\gamma$-phase yttrium trihydride $\mathrm{YH}_{3-\delta}$," J. Appl. Phys. 86, 6798-6802 (1999).

${ }^{12} \mathrm{~S}$. J. Van Der Molen et al., "Hydriding kinetics of Pd capped $\mathrm{YH}_{\mathrm{x}}$ switchable mirrors," J. Appl. Phys. 86, 6107-6119 (1999).

${ }^{13} \mathrm{~K}$. Wang, J. R. Hattrick-Simpers, and L. A. Bendersky, "Phase transformation in an yttrium-hydrogen system studied by TEM,” Acta Mater. 58, 2585-2597 (2010).

${ }^{14} \mathrm{M}$. W. Lee, C. Y. Kuo, H. C. Lin, and H. C. Wang, "Variations of the infrared transmission properties with the metal-insulator transition in thin films of the yttrium-hydride system," J. Appl. Phys. 89, 6135-6138 (2001).

${ }^{15}$ E. S. Kooij, A. T. M. Van Gogh, D. G. Nagengast, N. J. Koeman, and R. Griessen, "Hysteresis and the single-phase metal-insulator transition in switchable $\mathrm{YH}_{\mathrm{x}}$ films," Phys. Rev. B: Condens. Matter Mater. Phys. 62, 10088-10100 (2000).

${ }^{16}$ D. J. Santjojo, T. Aizawa, and S. Muraishi, "Ellipsometric characterization on multi-layered thin film systems during hydrogenation," Mater. Trans. 48, 1380-1386 (2007).

${ }^{17} \mathrm{O}$. V. Braginsky et al., "Removal of amorphous C and Sn on Mo:Si multilayer mirror surface in hydrogen plasma and afterglow," J. Appl. Phys. 111, 093304 (2012).

${ }^{18}$ D. Ugur, A. J. Storm, R. Verberk, J. C. Brouwer, and W. G. Sloof, "Kinetics of reduction of a $\mathrm{RuO}_{2}(110)$ film on $\mathrm{Ru}(0001)$ by atomic hydrogen," Microelectron. Eng. 110, 60-65 (2013).
${ }^{19}$ J. Rubio-Zuazo et al., "The multipurpose X-ray diffraction end-station of the BM25B-SpLine synchrotron beamline at the ESRF," Nucl. Instrum. Methods Phys. Res. Sect. A 716, 23-28 (2013).

${ }^{20}$ J. N. Huiberts et al., "Synthesis of yttrium trihydride films for ex-situ measurements," J. Alloys Compd. 239, 158-171 (1996).

${ }^{21}$ K. L. Kostov, W. Widdra, and D. Menzel, "Hydrogen on Ru (001) revisited: Vibrational structure, adsorption states, and lateral coupling," Surf. Sci. 560, 130-144 (2004).

${ }^{22}$ K. A. Gschneidner, "Physical properties of the rare earth metals," Bull. Alloy Phase Diagrams 11, 216-224 (1990)

${ }^{23} \mathrm{~V}$. V. Akhachinskij, "Enthalpy of formation of YH1.994 and YH2.792." in Proceedings of an International Symposium on Thermodynamics of Nuclear Materials (International Atomic Energy Agency, 1980), Vol. II.

${ }^{24}$ D. Khatamian, C. Stassis, and B. J. Beaudry, "Location of deuterium in $\alpha$-yttrium," Phys. Rev. B 23, 624-627 (1981).

${ }^{25}$ I. S. Anderson, J. J. Rush, T. Udovic, and J. M. Rowe, "Hydrogen pairing and anisotropic potential for hydrogen isotopes in yttrium," Phys. Rev. Lett. 57, 2822-2825 (1986).

${ }^{26}$ J. I. Langford and A. J. C. Wilson, "Scherrer after sixty years: A survey and some New results in the determination of crystallite size," J. Appl. Crystallogr. 11, 102-113 (1978).

${ }^{27}$ H. Oizumi, A. Izumi, K. Motai, I. Nishiyama, and A. Namiki, "Atomic hydrogen cleaning of surface $\mathrm{Ru}$ oxide formed by extreme ultraviolet irradiation of Ru-capped multilayer mirrors in $\mathrm{H}_{2} \mathrm{O}$ ambience," Jpn. J. Appl. Phys., Part 2 46, L633-L635 (2007).

${ }^{28}$ A. T. M. van Gogh, "Probing the metal-insulator transition in rare-earth based switchable mirrors," Ph.D. thesis (Vrije Universiteit, 2001).

${ }^{29} \mathrm{P}$. Hones, T. Gerfin, and M. Grätzel, "Spectroscopic ellipsometry of $\mathrm{RuO}_{2}$ films prepared by metalorganic chemical vapor deposition," Appl. Phys. Lett. 67, 3078 (1995).

${ }^{30} \mathrm{G}$. E. Jellison and F. A. Modine, "Parameterization of the optical functions of amorphous materials in the interband region," Appl. Phys. Lett. 69, 371-373 (1996).

${ }^{31}$ I. Nishiyama et al., "Reduction of oxide layer on $\mathrm{Ru}$ surface by atomic-hydrogen treatment," J. Vac. Sci. Technol. B 23, 3129 (2005).

${ }^{32}$ B. A. Belyaev and V. V. Tyurnev, "Electrodynamic calculation of effective electromagnetic parameters of a dielectric medium with metallic nanoparticles of a given size," J. Exp. Theor. Phys. 127, 608-619 (2018).

${ }^{33} \mathrm{X}$. Fan, W. Zheng, and D. J. Singh, "Light scattering and surface plasmons on small spherical particles," Light: Sci. Appl. 3, el79 (2014).

${ }^{34}$ C. F. Bohren and D. R. Huffman, Absorption and Scattering of Light by Small Particles (Wiley-Interscience, 1998). 